\title{
Interdiffusion and surface segregation in stacked self-assembled InAs/GaAs quantum dots
}

\author{
B. Lita and R. S. Goldman ${ }^{\text {a) }}$ \\ Department of Materials Science and Engineering, University of Michigan, Ann Arbor, \\ Michigan 48109-2136 \\ J. D. Phillips and P. K. Bhattacharya \\ Department of Electrical Engineering and Computer Science, University of Michigan, Ann Arbor, \\ Michigan 48109-2122
}

(Received 9 July 1999; accepted for publication 9 September 1999)

\begin{abstract}
We have investigated interdiffusion and surface segregation in molecular-beam-epitaxially-grown stacked self-assembled InAs/GaAs quantum dots. Using high-resolution cross-sectional scanning tunneling microscopy, we observe lateral variations in the vertical positions of In atoms in both the wetting layers and dot stacks. In some regions, the wetting layer thickness is much less than the dot height, while in other regions, the dot is immersed in the wetting layer. Using In and Ga atom counting, we obtain vertical In-Ga interdiffusion and 1/e segregation lengths of 1.25 and $2.8 \mathrm{~nm}$, respectively. In the dot stacks, significant In-Ga intermixing, primarily due to In surface segregation, is apparent. (C) 1999 American Institute of Physics. [S0003-6951(99)04244-8]
\end{abstract}

Self-assembled quantum dots have been shown to develop during epitaxial growth of highly mismatched films in the Stranski-Krastanow growth mode. ${ }^{1-3}$ After the initial growth of a few monolayers wetting layer, island nucleation results in the formation of self-assembled quantum dots. Stacks of capped quantum dots have the potential to form vertically ordered, three-dimensional (3D) dot lattices, which have unique electronic and optical properties. Although highly ordered arrays have been achieved in a number of materials systems, ${ }^{4}$ the limitations to the perfection of the dot lattices are not fully understood. In particular, cross-sectional transmission electron microscopy (XTEM) studies have shown that the dots are fully immersed in a wetting layer with thickness comparable to the dot height. ${ }^{5,6}$ On the other hand, thermal annealing has resulted in quenching of the photoluminescence emission associated with the wetting layer, suggesting that ideal 3D dot arrays may be achieved by controlled dissolution of the wetting layer. ${ }^{7}$ In order to achieve such ideal 3D arrays, an atomic level understanding of the regions between the dots and dot stacks is needed. Using ultrahigh vacuum (UHV) cross-sectional scanning tunneling microscopy (XSTM), we have mapped out the spa- tial distribution of In atoms in both the dot stacks and the wetting layers. The thickness of the wetting layer varies laterally such that it may be much less than or comparable to the dot height. Along the growth direction, the wetting layer is asymmetrically broadened. By counting the fraction of cation sites in the wetting layer occupied by In, we have directly determined the vertical $\mathrm{In}-\mathrm{Ga}$ interdiffusion and In surface segregation lengths. Significant vertical In-Ga intermixing in the dot stacks, primarily due to In surface segregation, is also apparent.

The stacked dot samples were grown by solid source molecular beam epitaxy, as described in a earlier report. ${ }^{8}$ The one- and five-layer dot stacks discussed here consisted of 2.6 monolayers of InAs and $5 \mathrm{~nm}$ GaAs, with a $10 \mathrm{~s}$ interrupt under $\mathrm{As}_{4}$ flux separating their growth at $510^{\circ} \mathrm{C}$. The oneand five-layer dot stacks were effectively in situ annealed at $620^{\circ} \mathrm{C}$ for 15 and $22.5 \mathrm{~min}$, respectively. For XSTM, the samples were cleaved to expose a ( $\overline{1} 10)$ surface, in an UHV chamber with base pressure $<5 \times 10^{-11}$ Torr. $^{8}$ All images were obtained with a constant tunnel current and sample bias voltages described below.

Figures 1(a), 1(b), 1(c), and 2(a) show high-resolution
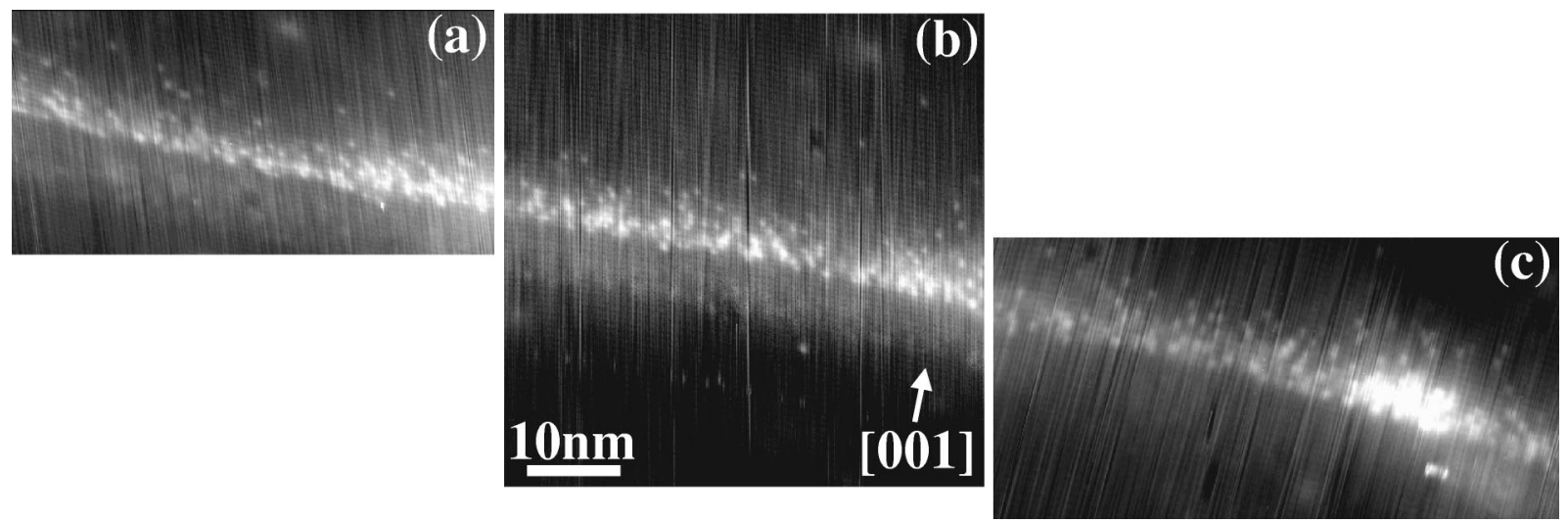

FIG. 1. High-resolution topographic images along a region of single layer dots acquired at sample bias voltage of $+2.2 \mathrm{~V}$. The gray-scale ranges displayed are (a) 1.4, (b) 1.4, and (c) $2.3 \AA$. The curvature of the wetting layer is an artifact due to piezoelectric creep.

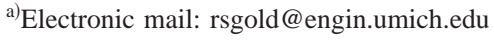




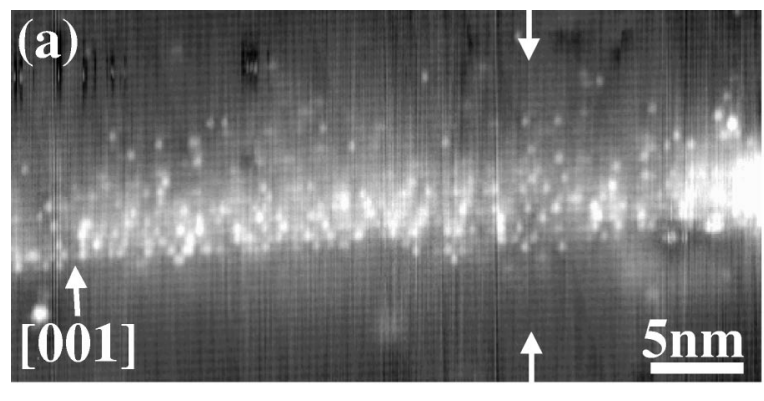

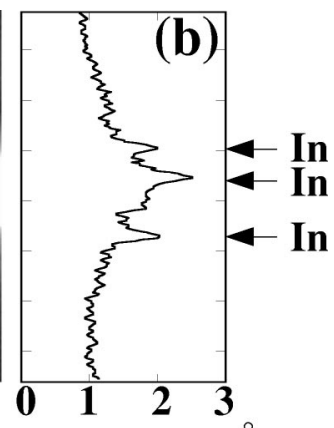

Tip Height $(\AA)$
FIG. 2. (a) Atomic-resolution topographic image of the wetting layer obtained at a sample bias of $+2.4 \mathrm{~V}$. The gray-scale range displayed is $2.4 \AA$. A cut along the line indicated by arrows in (a) is displayed in (b). topographic images along one-layer dots. In the darker regions of these images, fringes with a spacing of $5.65 \AA$, corresponding to the (001) lattice planes of GaAs are observed. Since these images were acquired with a positive sample bias voltage (empty state images), the cation sublattice is resolved and the bright spots correspond to In atoms in a GaAs matrix. ${ }^{9,10}$ Figures 1 (a) and 1(b) contain regions of a wetting layer, while Fig. 1(c) contains a bright oval region corresponding to an InAs dot. Similarly, Fig. 2(a) displays a wetting layer with part of a quantum dot in the far right. From these and many similar images, we observe that the fraction of sites occupied by In atoms in the wetting layer varies both vertically and laterally along the [001] and [110] directions, respectively. In some regions, such as Figs. 1(a) and 1(b), the wetting layer thickness is much less than the dot height. In other regions, such as Figs. 1(c) and 2(a), the dot is apparently immersed in the wetting layer. This is in contrast to other observations made by XTEM, where the dots seemed to be fully immersed in a 5-10 bilayers thick wetting layer. ${ }^{5,6}$ However, in XTEM, the thinnest regions of the wetting layer are unlikely to be detected due to the averaging through the foil thickness inherent in TEM.

In order to quantify the In profile along the growth di-
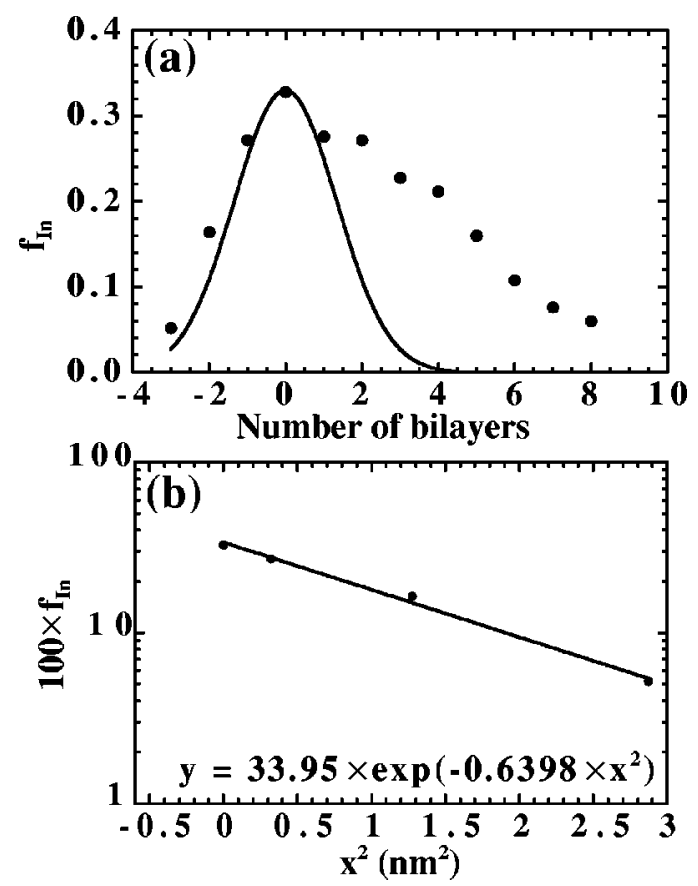

FIG. 3. (a) Fraction of sites occupied by In, $f_{\text {In }}$, as a function of the number of bilayers along the growth direction. (b) The concentration of In atoms, $C_{\text {In }}=100 \times f_{\text {In }}$, on a logarithmic scale vs distance squared, fit with a straight line. rection, we have performed In-Ga atom counting ${ }^{9,10}$ in many atomic resolution images such as those in Fig. 2(a). In particular, we determined the fraction of sites within each bilayer which are occupied by In atoms. In Fig. 2(b), a line cut of the tip height defined by the arrows in Fig. 2(a) contains sharp peaks, corresponding to individual In atoms, superposed on a high (spatial) frequency periodicity corresponding to the (001) planes of GaAs. In Fig. 3(a), the fraction of sites occupied by In, $f_{\text {In }}$, is plotted as a function of the number of bilayers, with the origin set artificially at that layer with the largest $f_{\text {In }}$. It is evident from the plot that $f_{\text {In }}$ is not symmetrical about the origin. Left of zero, $f_{\text {In }}$ apparently fits a gaussian, the thin film solution of the diffusion equation. Taking the concentration of In atoms as $C_{\mathrm{In}}=100$ $\times f_{\text {In }}$, we infer that $C_{\operatorname{In}} \propto \exp \left(-x^{2} / 4 D t\right)$, where $x$ is the distance of diffusion, $D$ is the diffusion coefficient, and $t$ is the time. We use the convention that the diffusion length is equal to $\sqrt{4 D t}$. In order to extract the In-Ga interdiffusion length, we plot $C_{\text {In }}$ on a logarithmic scale versus distance squared, and fit it with a straight line, such that the diffusion length is equal to the square root of the negative inverse of the exponent prefactor, $1.25 \mathrm{~nm}$. For comparison, we used our annealing time and temperature to calculate the diffusion length based upon diffusion coefficients obtained from annealing-induced shifts in photoluminescence emission of $\mathrm{In}_{0.5} \mathrm{Ga}_{0.5} \mathrm{As} / \mathrm{GaAs}^{11}$ and $\mathrm{In}_{0.67} \mathrm{Ga}_{0.33} \mathrm{As} / \mathrm{In}_{0.53} \mathrm{Ga}_{0.47} \mathrm{As}^{12}$ quantum wells. Since photoluminescence data requires detailed modeling for interpretation, it is not surprising that the calculated diffusion lengths, $2.74 \mathrm{~nm}^{11}$ and $0.79 \mathrm{~nm},{ }^{12}$ differ from the diffusion length determined here by direct atom counting.

In the plot of Fig. 3(b), a tail in $f_{\text {In }}$ is observed along the growth direction, probably due to surface segregation of In during growth. ${ }^{13}$ Assuming that $f_{\text {In }}$ decreases exponentially along the growth direction, ${ }^{14}$ the distance at which $f_{\text {In }}$ decreases to $1 / e$ of its initial value (the 1/e segregation length), is $2.8 \mathrm{~nm}$. This value is similar to a number of 1/e segregation lengths reported for similar growth conditions. ${ }^{14,15}$ However, a few groups have reported larger ${ }^{16}$ or smaller ${ }^{17,18} 1 / e$ segregation lengths, presumably due to differences in growth conditions and characterization methods which may be limited by averaging effects.

In stacked quantum dots, vertical In-Ga intermixing often occurs across the InAs dot/GaAs spacer layer interface near the center of the stack. Figure 4(b) shows line cuts of the tip height, $\mathrm{A}$ and $\mathrm{B}$, through the center and edge of a five-layer stack, defined by the arrows in Fig. 4(a). Each line cut has three distinct features: (1) a significant decrease in 


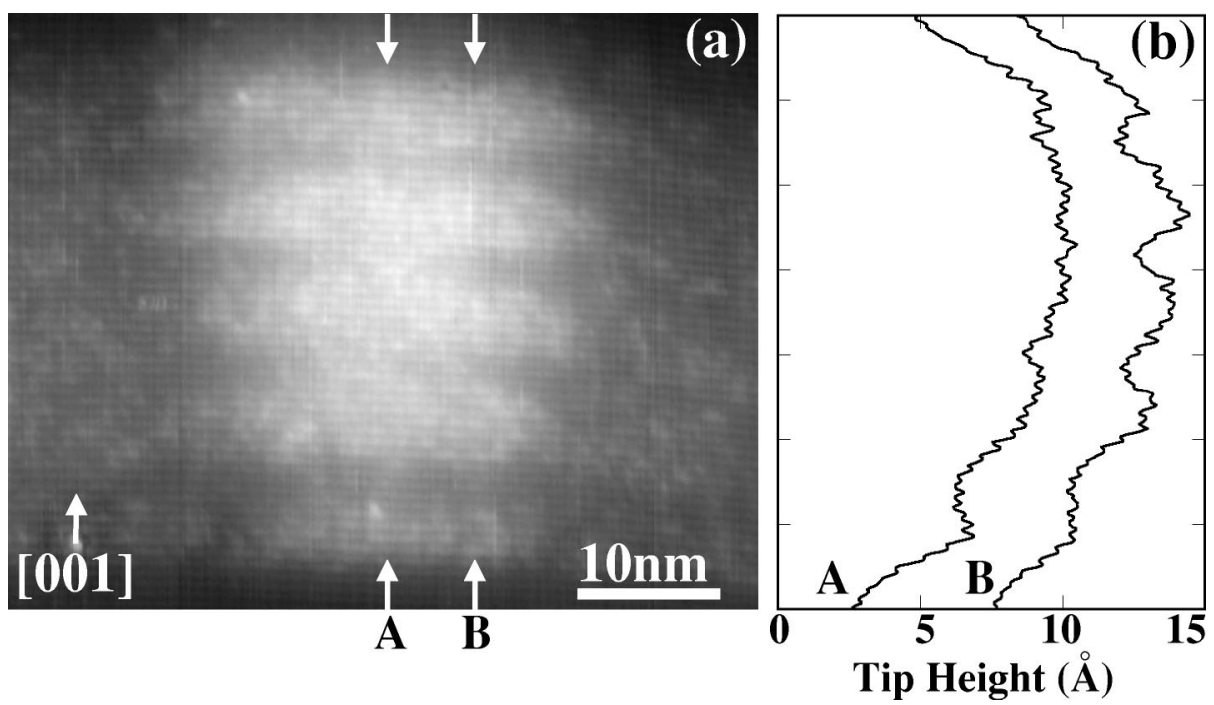

FIG. 4. (a) High-resolution topographic image of a five-layer stack of InAs/GaAs quantum dots, obtained at sample bias voltage of $-2.5 \mathrm{~V}$. The gray-scale range displayed is $7.3 \AA$ A. Cuts along the lines indicated by arrows $\mathrm{A}$ and $\mathrm{B}$ in (a) are displayed in (b).

the tip height outside of the dot columns due to surface distortions, (2) a high (spatial) frequency periodicity corresponding to the (001) planes of GaAs and InAs, and (3) a lower (spatial) frequency periodicity corresponding to the InAs dot/GaAs spacer layer. The distortion of the cleaved surface is reminiscent of the lateral tip height variations reported in earlier XSTM and cross-sectional atomic force microscopy studies of strain-compensated InGaAsP/InGaP superlattices. ${ }^{19,20}$ In that case, the cleaved surface distortion was attributed to elastic relaxation of strain resulting from a combination of lateral variations in alloy composition and layer thickness of the ternary and quaternary alloys. However, in our case, the distortion is due primarily to elastic relaxation of the compressive strain in the InAs dot stacks, similar to a recent report. ${ }^{21}$ Since the In concentration in the wetting layer is quite low, we expect that such surface distortions have not affected the accuracy of our wetting layer $\mathrm{In} / \mathrm{Ga}$ atom counting.

At the center of line cut A, the lower frequency periodicity is smeared out, indicating significant In segregation and/or In-Ga interdiffusion in the center of the dot column. It is interesting to note that the center of the dot column is also the position of highest strain. Hence, the In segregation and/or In-Ga interdiffusion is significantly enhanced by strain. The presence of significant In-Ga intermixing is further supported by a power spectral density analysis (not shown) of the high (spatial) frequency periodicity which indicates the presence of (001) planes of both InAs and GaAs for line cut B, but only those of InAs for line cut A. The extent of vertical intermixing in the dot stacks is often larger than the sum of the diffusion and segregation lengths discussed earlier. In addition, indium atoms are often observed on top of the last dot in a dot stack, but none are observed below the first dot in a stack. Hence, we expect that In surface segregation is the dominant mechanism of the vertical coupling between the stacked quantum dots. It is interesting to note that this vertical intermixing was not observed in an earlier study of stacked InAs/GaAs quantum dots grown at $485^{\circ} \mathrm{C},{ }^{22}$ presumably because of reduced In surface segregation occurring at the lower growth temperature. ${ }^{17}$

This work was supported in part by the Office of the Provost, the Office of the Vice President for Research, and the Rackham Graduate School, at the University of Michigan, the donors of the Petroleum Research Fund, administered by the American Chemical Society, the Dow Corning Foundation, the National Science Foundation (Grant Nos. DMR-9733707 and ECS-9628973), and the Army Research Office (Grant No. DAAG55-97-1-0156).

${ }^{1}$ I. N. Stranski and Von L. Krastanow, Akad. Wiss. Lit. Mainz. Abh. Math. Naturwiss. Kl. 146, 797 (1939).

${ }^{2}$ P. R. Berger, K. Chang, P. Bhattacharya, J. Singh, and K. K. Bajaj, Appl. Phys. Lett. 53, 684 (1988).

${ }^{3}$ D. Leonard, M. Krishnamurthy, C. M. Reaves, S. P. Denbaars, and P. M. Petroff, Appl. Phys. Lett. 63, 3203 (1993).

${ }^{4}$ G. Springholtz, V. Holy, M. Pinczolits, and G. Bauer, Science 282, 734 (1998), and references therein.

${ }^{5}$ P. D. Siverns, S. Malik, G. McPherson, D. Childs, C. Roberts, R. Murray, and B. A. Joyce, Phys. Rev. B 58, 10127 (1998).

${ }^{6}$ U. Woggon, W. Langbein, J. M. Hvam, A. Rosenauer, T. Remmele, and D. Gerthsen, Appl. Phys. Lett. 71, 377 (1997).

${ }^{7}$ R. Leon, Yong Kim, C. Jagadish, M. Gal, J. Zou, and D. J. H. Cockayne, Appl. Phys. Lett. 69, 1888 (1996).

${ }^{8}$ B. Lita, R. S. Goldman, J. D. Phillips, and P. K. Bhattacharya, Appl. Phys. Lett. 74, 2824 (1999).

${ }^{9}$ J. F. Zheng, J. D. Walker, M. B. Salmeron, and E. R. Weber, Phys. Rev. Lett. 72, 2414 (1994).

${ }^{10}$ M. Pfister, M. B. Johnson, S. F. Alvarado, H. W. M. Salemink, U. Marti, D. Martin, F. Morier-Genoud, and F. K. Reinhart, Appl. Phys. Lett. 67, 1459 (1995).

${ }^{11}$ R. Leon, D. R. M. Williams, J. Krueger, E. R. Weber, and M. R. Melloch, Phys. Rev. B 56, 4336 (1997).

${ }^{12}$ S. S. Rao, W. P. Gillin, and K. P. Homewood, Phys. Rev. B 50, 8071 (1994).

${ }^{13}$ J. Massies, F. Turco, A. Saletes, and J. P. Contour, J. Cryst. Growth 80, 307 (1987).

${ }^{14}$ J.-M. Gerard and J.-Y. Marzin, Phys. Rev. B 45, 6313 (1992).

${ }^{15}$ K. Muraki, S. Fukatsu, Y. Shiraki, and R. Ito, J. Cryst. Growth 127, 546 (1993).

${ }^{16}$ M. D. Williams, T. H. Chiu, and F. G Storz, J. Vac. Sci. Technol. B 13, 692 (1995).

${ }^{17}$ K. Radhakrishnan, S. F. Yoon, R. Gopalakrishnan, and K. L. Tan, J. Vac. Sci. Technol. A 12, 1124 (1994).

${ }^{18}$ Y. C. Kao, F. G. Celli, and H. Y. Liu, J. Vac. Sci. Technol. B 11, 1023 (1993).

${ }^{19}$ R. S. Goldman, R. M. Feenstra, C. Silvenius, B. Stålnacke, and G. Landgren, J. Vac. Sci. Technol. B 15, 1027 (1997).

${ }^{20} \mathrm{H}$. Chen, R. M. Feenstra, R. S. Goldman, C. Silvenius, and G. Landgren, Appl. Phys. Lett. 72, 1727 (1998).

${ }^{21} \mathrm{H}$. Eisele, O. Flebbe, T. Kalka, and M. Dahne-Prietsch, Surf. Interface Anal. 27, 537 (1999).

${ }^{22}$ W. Wu, J. R. Tucker, G. S. Solomon, and J. S. Harris, Jr., Appl. Phys. Lett. 71, 1083 (1997). 